\title{
Medikamentöse Behandlung von Menschen mit Persönlichkeitsstörungen in der forensischen Nachsorge
}

\author{
Tatjana Voß ${ }^{1} \cdot$ Daniela Calvano' $^{1}$ Joanna Vogel ${ }^{1}$ \\ Eingegangen: 9. Juni 2021 / Angenommen: 20. Juni 2021 / Online publiziert: 21. Juli 2021 \\ (c) Der/die Autor(en) 2021
}

\section{Zusammenfassung}

Der vorliegende Artikel präsentiert empirische Daten sowie drei Fallbeispiele zur medikamentösen Behandlung von Menschen mit Persönlichkeitsstörungen und komorbiden Störungen in der forensischen Nachsorge. In einer Studie wurden Daten von insgesamt 117 Patienten der Forensisch-Therapeutischen Ambulanz in Berlin ausgewertet. Die Stichprobe zeigte mit $60 \%$ eine hohe Prävalenz von Persönlichkeitsstörungen, wobei die dissoziale und die narzisstische Persönlichkeitsstörung bei Weitem den größten Anteil stellten. Gehäufte Komorbiditäten bestanden für Suchterkrankungen und Paraphilien. Die Personen, bei denen die Diagnose einer Persönlichkeitsstörung vorlag, wurden in $60 \%$ der Fälle medikamentös behandelt. In Bezug auf die Dauer der Therapie bis zum Stichtag im Dezember 2020 sowie die Anzahl der Krisen und Weisungsverstöße im Jahr 2020 ließen sich keine signifikanten Unterschiede im Vergleich zu Patienten mit anderen Störungsbildern feststellen. Die drei Fallberichte beschreiben Personen, die Sexualstraftaten begangen haben. Für sie bedeutete die kombinierte psychopharmakologische Behandlung aus Antiandrogenen und Psychopharmaka eine wirksame Unterstützung im Rahmen des spezifischen Gesamtbehandlungsplans in der forensischen Nachsorge. Die erhobenen Daten und Fallberichte werden hinsichtlich ihrer praktischen Relevanz für die Arbeit in forensischen Ambulanzen diskutiert.

Schlüsselwörter Persönlichkeitsstörungen $\cdot$ Paraphilien $\cdot$ Forensische Nachsorge $\cdot$ Medikation $\cdot$ Komorbiditäten Sexualstraftäter

\section{Drug treatment in forensic aftercare for patients diagnosed with personality disorders}

\begin{abstract}
This article presents empirical data and three case reports on pharmacological treatment in forensic aftercare of subjects diagnosed with personality disorders and comorbid psychiatric diagnoses. Data from 117 patients of the forensic outpatient clinic in Berlin were examined. Overall we found a prevalence of almost $60 \%$ of personality disorders in this sample, with antisocial and narcissistic personality disorder being the most common. Increased comorbidities were found for substance abuse disorders and paraphilic disorders in this sample. Of those diagnosed with personality disorder, $60 \%$ are prescribed a pharmacological treatment. No significant differences were found with regard to the duration of treatment up to the reference date in December 2020 and the number of crises and violations of conduct for the year 2020 for patients with a personality disorder compared to patients with other psychiatric disorders. The three case reports describe individuals who have committed sexual offenses and for whom a combined psychopharmacological treatment of antiandrogens and additional pharmacological medication as part of an overall treatment plan for forensic follow-up represented an effective intervention. The data and case reports are discussed with regard to their practical relevance for work in forensic aftercare.
\end{abstract}

Keywords Personality disorders $\cdot$ Paraphilic disorders $\cdot$ Forensic aftercare $\cdot$ Medication $\cdot$ Sexoffender Comorbidities

Dr. med. Tatjana Voß

tatjana.voss@charite.de

1 Institut für Forensische Psychiatrie,

Forensisch-Therapeutische Ambulanz, Charité -

Universitätsmedizin Berlin, Seidelstraße 38, 13507 Berlin,

Deutschland

\section{Einleitung}

Nach den aktuellen Qualitätskriterien (Freese und SchmidtQuernheim 2014; Schwarze et al. 2018) sollte forensische Nachsorge störungsspezifisch und individuell sowie 
multiprofessionell in einem Netzwerk der verschiedenen beteiligten Akteure erfolgen. Die Empfehlungen richten sich insbesondere an psychologische und ärztliche Psychotherapeut*innen, Ärzt*innen und Sozialpädagog*innen, die in forensischen Ambulanzen ehemalige Straffällige mit Psychosen, Paraphilien und Persönlichkeitsstörungen sowie Suchterkrankungen gemeinsam behandeln, begleiten und unterstützen.

In den Nachsorgeambulanzen nach Maßregelvollzug stellt die medikamentöse Behandlung der ehemaligen Straffälligen die Regel dar. Klassischerweise handelt es sich hier um Patienten mit schizophrenen Psychosen, die zur Rezidivprophylaxe und Verbesserung der Kriminalprognose meist dauerhaft neuroleptisch behandelt werden (Witzel 2016). Demgegenüber ist die medikamentöse Therapie in Ambulanzen des Strafvollzugs nicht oder noch nicht die Regel. Hier werden vor allem psychotherapeutische Behandlungen für Menschen mit Persönlichkeitsstörungen und zusätzlichen Komorbiditäten aus dem Bereich der paraphilen Störungen sowie Suchterkrankungen angeboten.

Für Patienten oder Klienten mit medikamentösem Therapiebedarf ist häufig eine zusätzliche externe fachärztliche Unterstützung erforderlich, um psychopharmakologische Behandlungen einzuleiten, durchzuführen und sicherzustellen. Dies ist beispielsweise von Relevanz bei Patienten mit einer Kombination aus Paraphilie und Persönlichkeitsstörung, bei denen gemäß der aktuellen Leitlinie der World Federation of Societies of Biological Psychiatry (WFSBP) 2020 eine medikamentöse Behandlung - z.B. mit einem Serotonin-Wiederaufnahmehemmer (SSRI) oder einem Antiandrogen (LHRH-Antagonist bzw. LHRH-Analogon) - indiziert ist. Auch bei Persönlichkeitsstörungen mit weiteren Komorbiditäten wie beispielsweise affektiven Störungen ist die individuelle Überprüfung und Durchführung einer psychopharmakologischen Behandlung im Einzelfall eine wichtige Maßnahme zur Verbesserung der Kriminalprognose und Verhinderung eines Rückfalls in erneute Straffälligkeit.

\section{Persönlichkeitsstörungen}

Nach ICD-10 und DSM-IV/DSM-5 werden Persönlichkeitsstörungen generell als meist früh in Kindheit und Jugend beginnende, anhaltende Muster von rigiden, nicht angepassten Denk- und Verhaltensweisen konzeptualisiert. Eine Persönlichkeitsstörung liegt dann vor, wenn infolge des Ausprägungsgrads oder der besonderen Konstellation von psychopathologisch relevanten Persönlichkeitszügen erhebliche subjektive Beschwerden und/oder nachhaltige Beeinträchtigungen in der sozialen Anpassung entstehen.

Persönlichkeitsstörungen beginnen bereits in der Adoleszenz oder im frühen Erwachsenenalter (Saß 1986). Bei ihrer Entstehung wirken unterschiedliche Faktoren zusammen, z. B. Temperament (Dynamik), Charakter (Struktur) sowie hormonelle und vegetative Funktionen in engem $\mathrm{Zu}$ sammenwirken mit Einflussfaktoren aus der Herkunftsfamilie, dem sozialen Umfeld, Erziehung und Bildung, Partnerschaftserfahrungen und kritischen Lebensereignissen ( $\mathrm{Sa}$ 2006). Die individuelle Persönlichkeit zeigt sich im Selbstbild und Wertgefüge, in der Intentionalität sowie in spezifischen Verhaltensmustern, Affektivität, Impulsregulation und Beziehungsgestaltung. Sie ist die Grundlage für subjektive Entscheidungs- und Begründungsmuster und wird deutlich in der Lebensgestaltung (Saß 2006).

Von Bedeutung sind in der forensischen Psychiatrie vor allem Persönlichkeitsstörungen des sogenannten Clusters B, die durch dramatische, emotional betonte, launische und impulsive Merkmale geprägt sind. Hierzu gehören die Borderline-Persönlichkeitsstörung, aber auch die histrionische, antisoziale und narzisstische Persönlichkeitsstörung.

Obwohl einzelne Symptome von Persönlichkeitsstörungen wie Angst, Impulsivität oder Unruhe klinischpsychopathologischen Auffälligkeiten anderer psychischer Störungsbilder der Achse 1 ähneln, liegen jedoch andere pathophysiologische Mechanismen zugrunde. Eine unbedingte Notwendigkeit zur dauerhaften medikamentösen Behandlung derartiger Symptome bei Menschen mit Persönlichkeitsstörungen kann von daher nicht generell postuliert werden. Vielmehr bleibt es sinnvoll, Achse-1-Störungen mit eher biologischem Ursprung von Achse-2-Störungen mit eher psychologischer Ätiologie klar voneinander zu differenzieren und hier auch nicht die unterschiedlichen Erfolgsraten für psychopharmakologische Behandlungen aus dem Auge zu verlieren (Koch et al. 2016).

Gleichwohl wird für verschiedene Symptome von Persönlichkeitsstörungen wie impulsiv-aggressive Verhaltensweisen auch in der forensischen Psychiatrie und Psychotherapie eine medikamentöse Behandlung durchaus in Betracht gezogen. Gerade bei schweren Persönlichkeitsstörungen scheint die Gabe von Psychopharmaka im Rahmen eines Gesamtbehandlungsplans erwägenswert und realisierbar. Allerdings handelt es sich jeweils um individuelle Entscheidungen im Off-label-Einsatz. Zielsymptome, Wirkung und Nebenwirkungen der entsprechenden Psychopharmaka (beispielsweise Neuroleptika) müssen hier besonders sorgfältig sowohl mit dem behandelten Patienten als auch im Helfernetzwerk festgelegt, überwacht und besprochen werden.

\section{Medikamentöse Behandlung von Persönlichkeitsstörungen}

Spezifische Psychopharmaka zur Behandlung der genannten Persönlichkeitsstörungen sind bislang nicht verfügbar 
(Benkert und Hippius 2021). Jede Therapie mit Psychopharmaka bei Persönlichkeitsstörungen bleibt somit ein individueller Behandlungsversuch (,off-label use“). Für die dissoziale/antisoziale Persönlichkeitsstörung liegen bislang überhaupt keine Medikationsempfehlungen vor. Zwar können einzelne Zielsymptome wie depressive oder andere affektive Symptome, unkontrollierbare Impulsivität und Aggressivität oder problematisches Verhalten pharmakologisch beeinflusst werden. Zur Behandlung von impulsiver Aggression bei antisozialen und psychopathischen Störungen existieren Wirksamkeitsnachweise für Fluoxetin, Phenytoin, Valproat, Carbamazepin und Lithium (Felthous et al. 2018). Neben einer häufig ablehnenden Haltung der Betroffenen gegenüber einer psychiatrischen Medikation, insgesamt hohen Abbruchraten und einer verstärkten Wahrnehmung unerwünschter Wirkungen ist bei einer solchen pharmakologischen Behandlung auch das Suizidrisiko zu berücksichtigen.

Optional können derzeit zur Behandlung von Persönlichkeitsstörungen aus der Medikamentengruppe der Antipsychotika Aripiprazol, Quetiapin und Olanzapin oder aus der Gruppe der Stimmungsstabilisatoren Valproat, Topiramat und Lamotrigin eingesetzt werden. Eine antidepressive Medikation kann bei vorherrschender Depressivität sowie komorbiden Angst- und Zwangsstörungen mit SSRI erfolgen. Weiterhin wird die Verordnung von Psychopharmaka zur Behandlung von Schlafstörungen, bei extremer innerer Anspannung, dissoziativen Symptomen, Alkoholabhängigkeit, ADHS und ausgeprägter therapierefraktärer Angst bei Menschen mit Persönlichkeitsstörungen empfohlen (Benkert und Hippius 2021).

Abb. 1 Anzahl der FTA-Patienten pro entsendender Einrichtung $(N=103$, Stand 12/2020)
Eine Besonderheit der forensischen Psychopharmakotherapie stellt die Behandlung von Persönlichkeitsstörungen mit komorbiden paraphilen Störungen dar. Verschiedene eingesetzte Präparate (Cyproteronacetat und LHRHAntagonisten sowie -Agonisten) führen dabei infolge der Absenkung des Testosteronspiegels auch zu einer Beruhigung impulsiver, dranghafter und aggressiver Persönlichkeitszüge.

\section{Untersuchung zu ehemaligen Straffälligen mit Persönlichkeitsstörungen in der FTA Berlin}

In der Berliner Forensisch-Therapeutischen Ambulanz (FTA) des Instituts für Forensische Psychiatrie an der Charité - Universitätsmedizin Berlin wurde im Februar 2021 untersucht, welche Auswirkungen eine psychopharmakologische Behandlung ehemaliger Straffälliger mit Persönlichkeitsstörungen auf den Verlauf der Nachsorgebehandlung hat. Gemäß den Vorgaben der beteiligten Berliner Senatsverwaltungen für Justiz und Gesundheit und der zugrundeliegenden Konzeption der FTA wurden die Probanden zur Hälfte aus dem Berliner Strafvollzug, inkl. der Abteilung für Sicherungsverwahrung, oder dem 63igerMaßregelvollzug zugewiesen. Die genaue Verteilung der entsendenden Einrichtungen kann Abb. 1 entnommen werden.

Laut Gesetz zur Führungsaufsicht hat die FTA die Aufgabe, einer verurteilten Person nach ihrer Entlassung aus dem Vollzug „helfend und betreuend“ zur Seite zu stehen und so

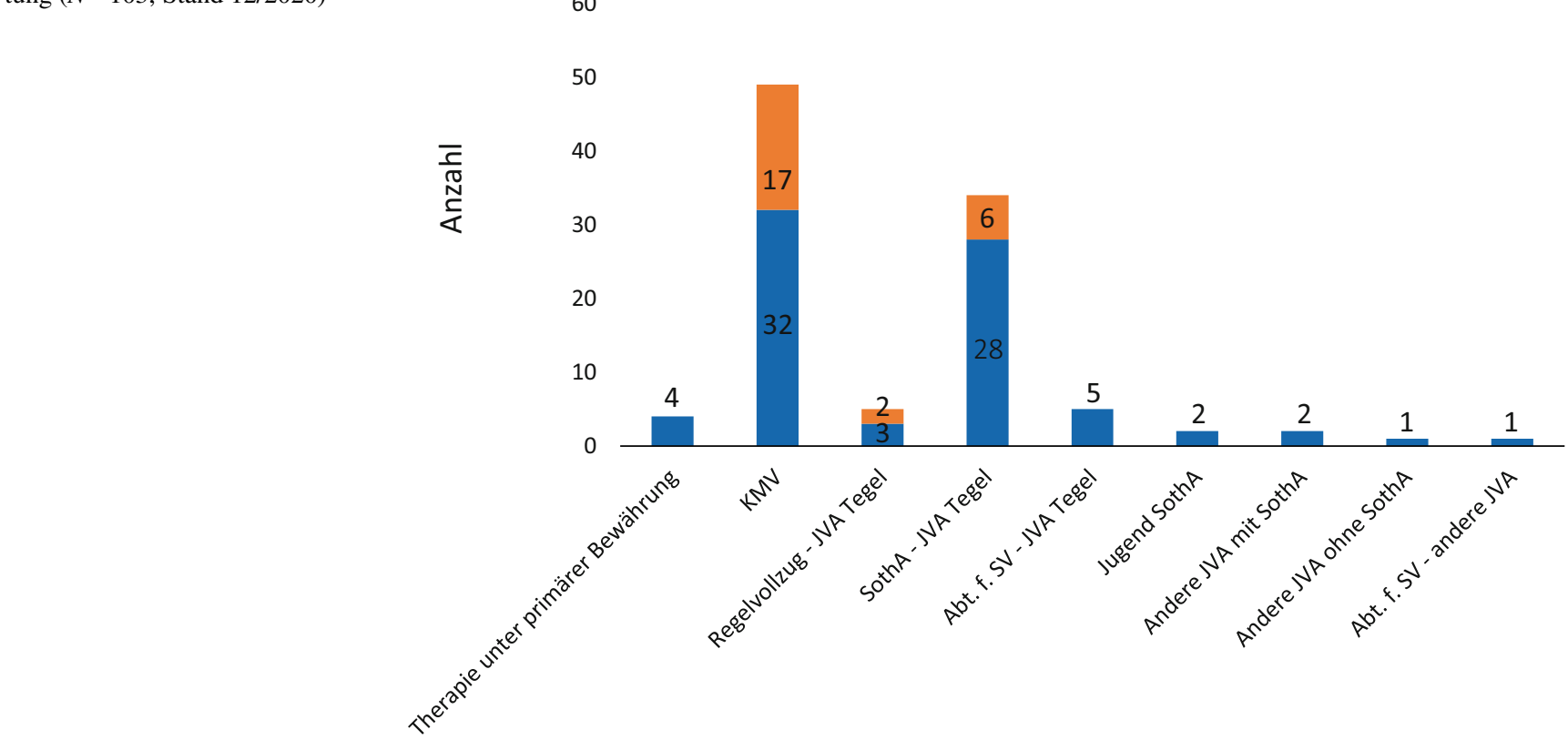


den im intramuralen Bereich erarbeiteten rückfallpräventiven Effekt strafrechtlicher Sanktionen oder forensischer Behandlung aufrechtzuerhalten. Die Nachsorgebehandlung erfolgt in multiprofessioneller Zusammenarbeit auf der Grundlage psychologischen wie psychiatrischen Fachwissens einschließlich einer medikamentösen Behandlung der Patienten mit paraphilen oder psychotischen Störungsbildern. Die Nachsorgebehandlung ist im Gegensatz zur intramuralen Behandlung in den zuweisenden Institutionen gekennzeichnet durch ein höheres Maß an Freiwilligkeit, geringeren rechtlichen Eingriffsmöglichkeiten sowie einen höheren Anteil an lebenspraktischen, alltagsbezogenen Inhalten.

In der aktuellen Untersuchung wurden die Daten von 117 langfristig in der FTA behandelten Patienten mit Persönlichkeitsstörungen hinsichtlich Krisen und Weisungsverstößen in Abhängigkeit von der psychopharmakologischen Behandlung erhoben und analysiert. Die Daten wurden der Basisdokumentation der FTA entnommen; die entsprechende Datenbank wird systematisch gepflegt und enthält kriminologische, kriminalprognostische und Behandlungsdaten (Erst- und Zweitdiagnose[n], Medikation). Darüber hinaus werden hier Krisen und Weisungsverstöße im Verlauf der Behandlung erfasst.

Die statistische Auswertung erfolgte mittels SPSS 26.0 für Windows (IBM Corporation 2011). Gruppenstatistische Vergleiche wurden mittels $t$-Tests für ungepaarte Stichproben berechnet. Das Alphalevel wurde auf $p<0,05$ für alle Tests festgesetzt.

\section{Häufigkeit von Persönlichkeitsstörungen}

Bei den insgesamt 117 Patienten bestand in vier Fällen eine organische Persönlichkeitsstörung als Erst- oder Zweitdia-

Tab. 1 Erst- und Zweitdiagnosen

\begin{tabular}{lll}
\hline Diagnose & Anzahl $(n)$ & Prozent $(\%)$ \\
\hline F0X & 4 & 3,4 \\
F1X.X & 38 & 32,5 \\
F20.X & 18 & 15,4 \\
F3X & 5 & 4,3 \\
F60.1 & 6 & 5,1 \\
F60.2 & 16 & 13,7 \\
F60.3 & 2 & 1,7 \\
F60.31 & 7 & 6,0 \\
F60.4 & 2 & 1,7 \\
F60.80 & 16 & 13,7 \\
F60.9 & 2 & 1,7 \\
F61 & 13 & 11,1 \\
F65.X & 50 & 42,7 \\
F60.X & 8 & 6,8 \\
$\sum$ & - & $100(\%)$ \\
\hline
\end{tabular}

Persönlichkeitsstörungen sind kursiv hervorgehoben gnose. Dreizehn Probanden hatten eine kombinierte oder andere Persönlichkeitsstörung (F61) als Erst- oder Zweitdiagnose, 51 Personen die Diagnose einer spezifischen Persönlichkeitsstörung. Demzufolge wurde bei insgesamt 68 von 117 Patienten (58,1\%) die Diagnose einer Persönlichkeitsstörung vergeben (Tab. 1). Am häufigsten kamen die dissoziale $(n=16 ; 13,7 \%)$ und narzisstische (ebenfalls $n=16 ; 13,7 \%)$ sowie die kombinierte Persönlichkeitsstörung $(n=13 ; 11,1 \%)$ vor, gefolgt von der schizoiden $(n=6$; $5,1 \%)$ und emotional instabilen Persönlichkeitsstörung vom Borderline-Typ $(n=7 ; 6,0 \%)$.

Bei einer Persönlichkeitsstörung als Erstdiagnose kamen die Patienten vermehrt aus dem Strafvollzug $(n=29$; $24,8 \%$ ) gegenüber 20 Patienten aus dem Maßregelvollzug $(17,9 \%)$. Lag hingegen eine Persönlichkeitsstörung als Zweitdiagnose vor, stammten die Patienten vermehrt aus dem Maßregelvollzug ( $n=12 ; 10,3 \%)$ gegenüber 7 Patienten aus dem Strafvollzug $(6,0 \%)$.

\section{Komorbiditäten}

In Tab. 2 ist die Häufigkeitsverteilung der Komorbiditäten bei den untersuchten Patienten dargestellt. Die häufigste Kombination ist die einer spezifischen oder kombinierten Persönlichkeitsstörung mit einer paraphilen Störung (als Erst- oder Zweitdiagnose mit jeweils $n=14$ bzw. $n=18$ Fällen, also insgesamt $n=32$ Fällen). Dies sind 47,1\% von insgesamt 68 Patienten mit einer Persönlichkeitsstörung.

Die zweithäufigste Kombination mit $n=14$ (12,0\%) ist die Erstdiagnose einer spezifischen Persönlichkeitsstörung mit der Zweitdiagnose einer psychischen und Verhaltensstörung durch psychotrope Substanzen. Diese Kombination findet sich auch bei weiteren 3 Probanden, wobei hier Erstund Zweitdiagnose umgekehrt gewichtet wurden. Somit finden sich insgesamt $n=17$ Fälle mit dieser Kombination, was $25,0 \%$ aller Patienten mit einer Persönlichkeitsstörung $(n=68)$ ausmacht.

Insgesamt haben nur 6 von 68 Patienten mit Persönlichkeitsstörung (8,8\%) keine Zweitdiagnose (Tab. 2).

\section{Medikation}

Von den Patienten, die eine Persönlichkeitsstörung als Erstoder Zweitdiagnose haben $(n=68)$, erhielten 41 Personen $(60,3 \%)$ ein oder mehrere Medikamente. Bei 16 Patienten $(23,5 \%)$ bestand aufgrund einer kombinierten paraphilen Störung eine antiandrogene Therapie oder eine hochdosierte SSRI-Behandlung. Weitere 10 Probanden $(14,7 \%)$ nahmen eine antidepressive Medikation ein, weitere 6 Personen $(8,8 \%)$ Neuroleptika. Eine stimmungsstabilisierende Medikation erhielten 11 Patienten $(16,2 \%)$. 
Tab. 2 Komorbiditäten von Persönlichkeitsstörungen

\begin{tabular}{|c|c|c|c|c|c|c|c|c|c|c|c|}
\hline \multirow[t]{2}{*}{ Erstdiagnose } & \multicolumn{11}{|c|}{ Zweitdiagnose } \\
\hline & $\overline{\text { F0X }}$ & F1X.X & F3X & $\mathrm{F} 4 \mathrm{X}$ & F60.X & F61 & F63.X & F65.X & F70.X & F8X & Keine \\
\hline F0X & 0 & 0 & 0 & 0 & 0 & 0 & 0 & 0 & 0 & 1 & 1 \\
\hline F1X.X & 0 & 1 & 0 & 0 & 3 & 0 & 0 & 0 & 0 & 0 & 0 \\
\hline F20.X & 0 & 7 & 0 & 0 & 0 & 0 & 0 & 2 & 0 & 0 & 5 \\
\hline F3X & 0 & 2 & 0 & 0 & 0 & 0 & 0 & 0 & 0 & 0 & 0 \\
\hline F60.X & 0 & 14 & 2 & 0 & 0 & 0 & 1 & 17 & 0 & 1 & 4 \\
\hline F61 & 2 & 4 & 0 & 0 & 0 & 0 & 0 & 1 & 0 & 0 & 1 \\
\hline F65.X & 0 & 6 & 0 & 1 & 9 & 5 & 1 & 0 & 2 & 4 & 10 \\
\hline F70.X & 0 & 2 & 4 & 0 & 0 & 0 & 0 & 5 & 0 & 0 & 1 \\
\hline
\end{tabular}

\section{Dauer der Behandlung}

Patienten mit einer organischen Persönlichkeitsstörung waren zum Zeitpunkt der Datenauswertung im Schnitt 87,6 Monate $(\mathrm{SD}=6,9)$ in Behandlung, das heißt zum Großteil auch über die Führungsaufsicht von 5 Jahren hinaus. Probanden mit einer spezifischen Persönlichkeitsstörung waren im Schnitt $\mathrm{M}=41,7$ Monate $(\mathrm{SD}=25,2)$ in Therapie, Personen mit einer kombinierten Persönlichkeitsstörung $\mathrm{M}=47,3$ Monate $(\mathrm{SD}=22,3)$. Zum Vergleich: Personen mit der Diagnose einer psychotischen Störung (ICD-10: F20.X) wurden im Schnitt $M=42,6$ Monate $(S D=30,3$ ) behandelt, Personen mit einer Intelligenzminderung (ICD10: $\mathrm{F} 70 . \mathrm{X}) \mathrm{M}=69,1$ Monate ( $\mathrm{SD}=22,1)$.

Vergleicht man die Behandlungsdauer bei allen Personen mit einer Persönlichkeitsstörung $\mathrm{M}=58,9(\mathrm{SD}=23,4)$ mit der Behandlungsdauer bei allen übrigen Patienten $(\mathrm{M}=48,2 ; \mathrm{SD}=26,1)$ wird jedoch deutlich, dass dieser Unterschied nicht signifikant ist $(\mathrm{T}(115)=-1,52 ; p=0,131)$.

\section{Gemeinsamkeiten und Unterschiede zwischen den Patientengruppen bezüglich Krisen und Weisungsverstöße}

Mittels t-Tests für ungepaarte Stichproben wurden $n=68$ Personen mit Persönlichkeitsstörungen mit den übrigen Patienten der FTA verglichen hinsichtlich Anzahl der Krisen sowie Anzahl der Weisungsverstöße jeweils im Jahr 2020. Patienten mit Persönlichkeitsstörung erlebten im Schnitt $\mathrm{M}=0,73 ; \mathrm{SD}=3,02$ Krisen, Patienten mit anderen Diagnosen $\mathrm{M}=0,62 ; \mathrm{SD}=1,33$ Krisen. Patienten mit Persönlichkeitsstörung produzierten im Schnitt $\mathrm{M}=1,49 ; \mathrm{SD}=2,02$ Weisungsverstöße, Patienten mit anderen Diagnosen $\mathrm{M}=2,13$; $\mathrm{SD}=1,98$ WeisungsverstöBe. Beide Analysen ergaben, dass es hinsichtlich dieser Merkmale keinerlei Unterschiede zwischen den Gruppen gibt (Krisen $\mathrm{T}(115)=-0,272 ; p=0,786$; Weisungsverstöße $\mathrm{T}(115)=-0,322 ; p=0,749)$.

Mittels t-Tests für ungepaarte Stichproben wurden nichtmedizierte $(n=33)$ und medizierte Patienten $(n=35)$ mit einer Persönlichkeitsstörung hinsichtlich der Anzahl der durchlebten Krisen sowie der begangenen Weisungsverstöße verglichen. Für das Jahr 2020 durchlebten nichtmedizierte Personen im Schnitt $\mathrm{M}=0,24$ Krisen $(\mathrm{SD}=0,61)$ gegenüber medizierten Personen mit im Schnitt M=1,14 Krisen $(\mathrm{SD}=4,13)$. Für das Jahr 2020 wurden bei nichtmedizierten Personen im Schnitt $\mathrm{M}=1,23$ ( $\mathrm{SD}=0,54)$ Weisungsverstöße gemeldet gegenüber $\mathrm{M}=1,74(\mathrm{SD}=0,38)$ bei medizierten Personen. Auch hier ergaben die Analysen, dass hinsichtlich dieser Merkmale keine Unterschiede zwischen den Gruppen bestehen (Krisen T(66) $=-1,04 ; p=0,322$; Weisungsverstöße $\mathrm{T}(66)=-1,02 ; p=0,235)$.

Im Folgenden soll anhand von drei Fallvignetten die ambulante Nachsorgebehandlung ehemaliger Straffälliger mit Persönlichkeitsstörung, die wegen Sexualstraftaten im Berliner Straf- und Maßregelvollzug zum Teil Jahrzehnte untergebracht waren, beschrieben werden. Bei allen drei untersuchten Personen bestand neben einer Persönlichkeitsstörung auch eine paraphile oder andere komorbide psychiatrische Störung. Zudem wurden alle drei über Jahre zusätzlich zu Antiandrogenen auch mit Psychopharmaka therapiert. Der Beitrag beschreibt die klinischen Erfahrungen, die mit der medikamentösen Behandlung dieser Straftäter gesammelt wurden, einschließlich ihrer Bedeutung und Konsequenzen für die forensische Nachsorge.

\section{Fallvignetten}

\section{Fallbeispiel 1: Herr A.}

Diagnosen:

- Emotional-instabile Persönlichkeitsstörung vom Borderline-Typ (F60.31)

- Pädophilie (F65.4)

Zur Biographie von Herrn A., 60 Jahre, ist zu vermerken, dass er kurz nach seiner Geburt in eine Pflegefamilie kam. Er war kleinwüchsig und seine frühkindliche Entwicklung verlief verzögert. Bereits in der Schulzeit fiel er 
durch Verhaltensauffälligkeiten wie Stören im Unterricht, schwere Wutanfälle und schlechte Schulleistungen auf, sodass mehrfach Klassenwiederholungen und Schulwechsel erfolgten. Er verließ die Schule mit einem Abgangszeugnis der 10. Klasse. Eine Lehre brach er nach einem Monat ab. Herr A. lebte von Gelegenheitsjobs und der finanziellen Unterstützung durch die Pflegemutter.

Bereits als Jugendlicher war er ein Einzelgänger und fühlte sich zu Kindern hingezogen. Im Alter von 22 Jahren erfolgte die erste Verurteilung wegen sexuellen Missbrauchs. Bis zu seiner Unterbringung lebte Herr A. sozial isoliert und konsumierte Alkohol und Drogen. Außer zu seiner Pflegemutter bestanden keine festen Beziehungen. Mit 23 Jahren erfolgten zwei Suizidversuche, mit 24 Jahren wurde er erstmalig inhaftiert. Es folgten bis zum Anlassdelikt drei weitere Verurteilungen wegen einschlägiger Delikte. Mit 32 Jahren wurde die Unterbringung in einem psychiatrischen Krankenhaus wegen sexuellen Missbrauchs eines Kindes angeordnet.

Der 25 Jahre dauernde Behandlungsverlauf im Maßregelvollzug war vor allem in den ersten Jahren durch erhebliche eigen- und fremdgefährdende Handlungen geprägt. Es kam zur Ingestion von Rasierklingen sowie Brandstiftung im Rahmen von Anspannungszuständen. In der psychotherapeutischen Arbeit zeigten sich ein niedriges Strukturniveau mit Störungen der Selbstwahrnehmung, Selbststeuerung, Objektwahrnehmung sowie einer Bindungsstörung.

Bezüglich seiner paraphilen Störung zeigte sich Herr A. therapiewillig. Seit 2004 wurde er durchgehend mit einem LHRH-Analogon behandelt. Der Behandlungsverlauf war jedoch entscheidend durch die Persönlichkeitsstörung geprägt. So kam es immer wieder zu Stimmungsschwankungen und einem raschen Frustrationserleben, worauf Herr A. mit sozialem Rückzug, Aufgabe der tagesstrukturierenden Maßnahmen und depressiven Symptomen reagierte. Hier verfiel er immer wieder in alte Verhaltensmuster mit Konsum von Drogen oder Missbrauchsabbildungen. Die medikamentöse Behandlung wurde daher um eine antidepressive Therapie mit Citalopram $40 \mathrm{mg}$ täglich ergänzt sowie um psychotherapeutische Elemente, wie z.B. eine Skillsgruppe.

Insgesamt zeigten sich dann im Verlauf der Behandlung eine Beruhigung und Nachreifung der Persönlichkeit. Stimmungsschwankungen, resignierendes Vermeidungsverhalten und Tendenzen zur kurzfristigen Bedürfnisbefriedigung wurden deutlich abgemildert. 2019 konnte Herr A. in ein hochstrukturiertes, kontrollierendes und unterstützendes ambulantes Helfernetzwerk entlassen werden. Die Ziele der Nachsorgebehandlung bestanden (bis zum aktuellen Zeitpunkt) in der Sicherstellung der triebdämpfenden und antidepressiven Medikation sowie der Alkohol- und Drogenabstinenz, dem engmaschigen Austausch des umfassenden Helfernetzwerks zur frühzeitigen Erfassung von Rückzugs- tendenzen des Patienten, der Sicherung einer nicht überfordernden Tagesstrukturierung sowie der Aufrechterhaltung der Aufmerksamkeit für die vorliegende Pädophilie.

Auch im Verlauf der Bewährung kam es wiederholt zu Situationen, in denen sich Herr A. überfordert fühlte, zu problematischem Verhalten mit sozialem Rückzug, Nichteinhalten von Absprachen und Drogenkonsum, was jedoch durch die wöchentliche forensische Nachsorge abgefangen werden konnte. Entsprechende Weisungsverstöße wurden mit der zuständigen Strafvollstreckungskammer kommuniziert. Krisen in Sinne von schweren depressiven Episoden, erhebliche eigen- oder fremdgefährdende Fehlhandlungen sowie deliktnahes Verhalten konnten seit der Entlassung aus der Klinik bislang nicht mehr festgestellt werden.

\section{Fallbeispiel 2: Herr B.}

\section{Diagnosen:}

- Organische Persönlichkeitsstörung mit emotional instabilen Zügen (F07.0)

- Lernbehinderung (F81.9)

- Epilepsie mit fokal eingeleiteten Anfällen (G40.9)

- Psychogene Anfälle (F44.5)

Der heute 35-jährige Herr B. wuchs als Einzelkind bei seiner alleinerziehenden Mutter auf. Diese litt selbst unter starken Stimmungsschwankungen und wird als impulsiv und stark aufbrausend geschildert. Zudem habe die Mutter viel Alkohol getrunken und sei darunter aggressiv geworden, sodass Herr B. vom 7. bis zum 14. Lebensjahr bei seiner Großmutter aufwuchs. Bereits in der zweiten Klasse einer Förderschule fiel er durch Konzentrationsstörungen, motorische Unruhe und gesteigerte Erregbarkeit mit aggressivem Verhalten auf. Im weiteren Verlauf kam es neben wiederholtem Einnässen zu zunehmenden oppositionellen Verhaltensweisen mit Lügen und häufigen Aggressionen, sodass die Großmutter an ihre Grenzen stieß und Herrn B. in ein Heim eingewiesen werden musste. Dennoch setzten sich die Verhaltensauffälligkeiten fort und Herr B. wechselte im Folgenden mehrfach das Heim.

Während seiner Heimunterbringung sei Herr B. durch einen Mitbewohner sexuell missbraucht worden. Er habe dann 14-jährig seinen späteren, langjährigen Missbrauchstäter kennengelernt. Dieser habe ihn beim Sport mehrmals zu sich nach Hause eingeladen. Er habe in dem Täter einen Vaterersatz gesehen. Im weiteren Verlauf kehrte Herr B. dann in seine Primärfamilie zurück.

Nach dem Hauptschulabschluss absolvierte Herr B. einen berufsbegleitenden Lehrgang, eine Ausbildung als Koch brach er jedoch ab. Bereits in seiner Jugend begann er, regelmäßig Cannabis zu konsumieren. Ab dem 21. Lebensjahr erfolgten mehrfache stationäre psychiatrische Behandlungen aufgrund depressiver Stimmungsein- 
brüche ohne nachhaltige psychische Stabilisierung. Bereits in den damaligen Epikrisen wurde eine Polypharmazie mit zahlreichen Medikamentenwechseln beschrieben. Die Persönlichkeit von Herrn B. zeichnete sich durch dramatische Selbstdarstellung, Distanzminderung, impulsives Handeln und Stimmungsschwankungen aus. Dabei war die sexuelle Identität des Patienten wenig ausgebildet; er führte vielfältige, zum Teil wahllose kurzfristige heteround homosexuelle Beziehungen.

Mit 22 Jahren beging Herr B. mehrfach sexuellen Missbrauch an Kindern und wurde zu einer Gesamtfreiheitsstrafe von vier Jahren verurteilt. Während der Inhaftierung nahm er am Gruppenprogramm der Sozialtherapeutischen Anstalt teil, musste im Verlauf jedoch aufgrund seiner Interaktionsschwierigkeiten aus dem Programm herausgenommen werden und erhielt im Folgenden Einzeltherapie.

Aufgrund paranoid anmutender Gedanken erhielt Herr B. eine antipsychotische Medikation mit Amisulprid, die bis zur Haftentlassung auf $1200 \mathrm{mg} / \mathrm{Tag}$ aufdosiert wurde. Zudem wurde der Verdacht auf eine Epilepsie gestellt und Herr B. erhielt eine antikonvulsive Medikation mit Carbamazepin. Im weiteren Verlauf stellte sich bei ihm eine ausgeprägte Polypharmazie ein. So erhielt er zwischenzeitlich bis zu vier Psychopharmaka gleichzeitig mit mehrfachen Umstellungen, und er benötigte weiterhin zusätzliche Schmerzmedikamente. Herr B. lehnte und lehnt eine antiandrogene Medikation grundsätzlich ab aufgrund der Befürchtung, dass bei einem Verzicht auf seine sexuellen Bedürfnisse das Leben für ihn nicht „lebenswert“ sei.

Nach der Haftentlassung wohnte Herr B. zunächst bei seiner Großmutter; erst nach längerer Zeit konnte er in eine therapeutische Wohngemeinschaft entlassen werden. Im weiteren Verlauf der forensischen Nachsorge kam es immer wieder zu krisenhaften Zuspitzungen. Bezüglich der Epilepsie erfolgte im Verlauf eine umfassende neurologische Abklärung inklusive Langzeit-EEG, in der neben einer fraglichen idiopathischen Epilepsie auch dissoziative Anfälle bestätigt werden konnten. Auf den Versuch, die antikonvulsive Medikation zu reduzieren, reagierte Herr B. mit einer Frequenzzunahme dissoziativer Anfälle, sodass auf seinen Wunsch hin davon im Verlauf wieder Abstand genommen wurde.

Trotz oder möglicherweise auch wegen einer zunehmenden psychischen Stabilisierung und eines gefestigten psychosozialen Netzwerks verstieß Herr B. in einem Sportverein gegen seine Kontaktweisung, was durch im Internet veröffentlichte Bilder von Herrn B. mit Jungen im Präferenzalter publik wurde. Die Führungsaufsichtsstelle stellte daraufhin einen Antrag auf Verlängerung der Führungsaufsicht über die fünf Jahre hinaus, dem die Strafvollstreckungskammer entsprach.

\section{Fallbeispiel 3: Herr C.}

Diagnosen:

- Lernbehinderung (F81.9)

- Kombinierte Persönlichkeitsstörung (F61.0)

- Pädophile Störung (F65.4)

Bei Herrn C., 51 Jahre, bestanden seit der frühen Kindheit Verhaltensauffälligkeiten wie Bettnässen und Zündeln; er zeigte sich aggressiv gegenüber anderen Kindern und hatte einen Sprachfehler. Als 7-Jähriger erfolgte die Heimunterbringung. Herr C. besuchte acht Klassen der Sonderschule, ehe er in einen Jugendwerkhof eingewiesen wurde. Mit 17 Jahren erfolgte die erste Inhaftierung nach einer erheblichen Anzahl von Einbruchdiebstählen, gemeinschaftlicher vorsätzlicher Beschädigung, Sachbeschädigung sowie Betrug in Tateinheit mit Urkundenfälschung. Kurz nach seiner Haftentlassung tötete Herr C. ein Kleinstkind und wurde wegen Mord und Vorbereitung zum Mord in Tateinheit mit Kindsentführung sowie sexuellem Missbrauch eines Kindes nach der Gesetzgebung der DDR zu 14 Jahren Freiheitsstrafe verurteilt.

Laut Gutachten im Erkenntnisverfahren ist Herr C. intellektuell grenzbegabt und zeigt eine schwerwiegende abnorme Fehlentwicklung der Persönlichkeit mit Krankheitswert. Er wird als erheblich affektlabil, frustrationsintolerant, paranoid, exzentrisch-egozentrisch, infantil beschrieben. In der anschließenden über 20-jährigen Unterbringung und Inhaftierung schloss Herr C. die Hauptschule und eine Lehre zum Gärtner ab. Es erfolgten eine medikamentöse Triebdämpfung mit Salvacyl sowie eine neuroleptisch-sedierende Behandlung mit Olanzapin $20 \mathrm{mg}$ und Amitriptylin $225 \mathrm{mg}$.

Nach der Entlassung aus der JVA war es zunächst vorrangiges Ziel der FTA, das erforderliche umfangreiche Helfersystem - betreute Wohneinrichtung, gesetzlicher Betreuer, Sozialpsychiatrischer Dienst, Bewährungshelfer - zu koordinieren und gemeinsame Behandlungsziele festzulegen. Herr C. zeigte sich nach über 20 Jahren Unterbringung durch die neue Situation in Freiheit anfänglich sehr belastet. So gelang eine berufliche Reintegration in eine Werkstatt für Menschen mit Behinderung erst nach der Integration in eine betreute Wohneinrichtung. Bis heute leidet Herr C. intermittierend unter Krisen mit Stimmungsschwankungen, Selbstvorwürfen und Schuldgefühlen und droht mit dem Abbruch der Behandlung bzw. bricht sie dann auch für Wochen ab.

Im Verlauf der Therapie wurde deutlich, dass es Herrn C. aufgrund der schweren Persönlichkeitsstörung schwerfällt, private Kontakte außerhalb des Helfersystems aufzubauen. Infolge der antiandrogenen und sedierenden Medikation bestehen bei Herrn C. zudem Nebenwirkungen in Form einer Reduktion der allgemeinen Belastbarkeit und der kognitiven 
Funktionen sowie in Form einer allgemeinen medikamentösen Dämpfung. Im Rahmen der umfassenden multiprofessionellen Betreuung und komplexen Begleitung konnten diese schwerwiegenden Defizite im Bereich der sozialen Fertigkeiten im Verlauf verbessert werden. Die sedierende neuroleptische und antidepressive Medikation wurde schrittweise abgesetzt bzw. nur noch in Krisensituationen als Bedarfsmedikation zur Reizabschirmung eingesetzt. Deliktnahes Verhalten konnte somit zwar reduziert, jedoch nicht gänzlich verhindert werden.

\section{Diskussion}

Zentrales Element der Therapie von straffälligen Menschen mit Persönlichkeitsstörungen im Straf- und Maßregelvollzug ist grundsätzlich die psychotherapeutische Behandlung. Forensische Psychotherapie kann dabei auch als erfolgreich angesehen werden, wenn die Symptomatik zwar nicht vollständig reduziert ist, die Entlassung des Patienten aus der Behandlungseinrichtung jedoch „kontrollierbar“ erscheint, beispielsweise im Rahmen einer effektiven forensischen Nachsorge (Lau et al. 2019). Wenn psychotherapeutische Ansätze allein jedoch die deliktrelevante Symptomatik nicht ausreichend bessern können und/oder forensisch relevante Komorbiditäten bestehen, stellt die pharmakologische Behandlung von Patienten mit Persönlichkeitsstörungen eine wichtige zusätzliche Therapieoption dar. Sie kann in einem multidisziplinären Behandlungsansatz die Kriminalprognose des Straftäters verbessern, ihn auf dem Weg zu seiner Entlassung unterstützen und seine Rehabilitation ermöglichen. Die Behandlung der jeweiligen Persönlichkeitsstörungen richtet sich an den für die Diagnosestellung relevanten Funktionseinbußen aus (Hauser et al. 2021).

Gerade für das Störungsbild der Borderline-Persönlichkeitsstörung existieren inzwischen eine Reihe von medikamentösen Therapieempfehlungen (Hancock-Johnson et al. 2017; Stoffers und Lieb 2015; Gunderson et al. 2018; Stoffers et al. 2010). Allerdings ist bisher noch für keine der klinisch erprobten Behandlungsansätze (SSRI, Neuroleptika der zweiten Generation, Phasenstabilisatoren) ein wissenschaftlich fundierter Evidenznachweis erfolgt. Wenn auch der fehlende Wirkungsnachweis kein Beleg für eine tatsächlich fehlende Wirksamkeit ist, bedarf es hier doch - gerade bei richterlicher Weisung zur Einnahme von Psychopharmaka in der Führungsaufsicht - einer besonders sorgfältigen Indikationsstellung mit Überprüfung von Wirkung und unerwünschten Wirkungen der Präparate (Howner et al. 2020).

Ehemals straffällige Menschen mit Borderline-Persönlichkeitsstörungen sind gefährdet, Opfer eines polypragmatischen bzw. polypharmazeutischen Behandlungsregimes zu werden. Eine sich im Laufe der Jahre gegebenenfalls auf- bauende Polypharmazie, bei der kein Versuch unternommen wird, wirkungslose Präparate abzusetzen, beeinträchtigt zum einen die Mitwirkungsfähigkeit der Patienten bei der notwendigen forensischen Rehabilitation. Zum anderen reduziert sich aufgrund der oft deutlichen Nebenwirkungen wie Sedierung und Gewichtszunahme die Fähigkeit der Betroffenen zur Teilhabe. Ein Reduktionsversuch bei einer solchen Polypharmazie kann ambulant in einem stabilen Helfernetzwerk erfolgen, das den Patienten aufmerksam begleitet, psychopathologische Veränderungen rechtzeitig bemerkt und rückmeldet bzw. unmittelbar intervenieren kann.

Im Alltag der Nachsorgebehandlung von Patienten mit Persönlichkeitsstörungen ist die Berücksichtigung von Komorbiditäten, vor allem aus dem Bereich der paraphilen Störungen, von besonderer Bedeutung. Die Berücksichtigung dieser Komorbiditäten im Rahmen eins individuellen Behandlungskonzeptes kann Rückfallrisiken bei Sexualstrafstörungen effektiv senken (Hörburger und Habermeyer 2020). Die antiandrogene Behandlung von Sexualstraftätern mit einer Persönlichkeitsstörung kann auch im ambulanten Setting nach WFSBP 2020 leitliniengemäß erfolgen. Sie stellt für diese Patientengruppe neben der Psychotherapie die zweite entscheidende Säule der Behandlung dar. Die Gabe von Antiandrogenen hat dabei das Ziel, den Testosteronwert bis in den Kastrationsbereich abzusenken. LHRHAnaloga zeigen, Berichten behandelter Patienten zufolge, eine zuverlässige Wirkung im Hinblick auf die Abnahme sexueller Fantasien, Interessen und Erregung (Turner und Briken 2018). Zwar ist die dauerhafte Behandlung mit Antiandrogenen mit einer Vielzahl möglicher Nebenwirkungen verbunden, wie beispielsweise Ermüdung, depressiver Stimmung, hepatozellulären Dysfunktionen, testikulärem Abbau, Gynäkomastie, Abnahme der Knochendichte, Nierensteinen und einem erhöhten Risiko für kardiovaskuläre Ereignisse. Dennoch scheinen Antiandrogene bei Menschen mit paraphilen Störungen und Persönlichkeitsstörungen durch die Senkung des Testosteronspiegels auch eine affektausgleichende Wirkung auf die Persönlichkeit mit verbesserter Ansprechbarkeit auf psycho- und soziotherapeutische Maßnahmen zu erreichen. Damit wird in der Folge insgesamt das Rückfallrisiko für erneute Sexualstraftaten gesenkt.

In der Berliner Forensisch-Therapeutischen Ambulanz weisen weit über die Hälfte der Patienten eine primäre oder komorbide Persönlichkeitsstörung auf. Am häufigsten finden sich die dissoziale und narzisstische Persönlichkeitsstörung, gefolgt von der Borderline-Persönlichkeitsstörung. Über die Hälfte der Patienten mit einer Persönlichkeitsstörung als Erst- oder Zweitdiagnose wird mit Psychopharmaka und/oder Antiandrogenen behandelt.

Die Auswertung der Daten von 117 Patienten mit Persönlichkeitsstörungen in der FTA Berlin ergab, dass die Betroffenen zur Hälfte mit Psychopharmaka behandelt 
werden. Die Ergebnisse zeigen zudem, dass die Probanden ebenso viele Krisen durchmachen und WeisungsverstöBe pro Jahr begehen wie Patienten mit anderen psychiatrischen Störungsbildern (z.B. Psychosen oder Intelligenzminderung). Die geschilderten drei Fallvignetten der ehemaligen Sexualstraftäter mit organischer und emotional instabiler Persönlichkeitsstörung machen deutlich, wie bei derartigen schweren Störungsbildern eine kombinierte pharmakologische Behandlung aus Antiandrogenen und Psychopharmaka im Rahmen des Gesamtbehandlungsplans eine wirksame Unterstützung darstellt. Erforderlich ist dabei die genaue, individuelle Analyse von Ressourcen und Stärken, aber auch von Grenzen und Defiziten der Patienten mit Persönlichkeitsstörung.

Neben der psychotherapeutischen Behandlung profitierten die Betroffenen auch von der zusätzlichen Pharmakotherapie, da sie hierunter weniger impulsiv, depressiv oder resignativ Krisen durchlitten und/oder in geringerem Maße mit Weisungsverstößen reagierten. Auch konnten sie psychotherapeutische Interventionen besser umsetzen und die forensische Nachsorgebehandlung erfolgreich absolvieren, ohne erneut einschlägig rückfällig zu werden. Die Zufriedenheit von Behandlern und Behandelten stieg dabei auch mit der verbesserten Gestaltung des Umgangs und der Kommunikation miteinander, sowohl im Hilfenetzwerk als auch im Behandlungsteam.

Die statistische Auswertung der genannten Daten zeigt, dass auch medikamentös behandelte Patienten mit Persönlichkeitsstörungen Weisungsverstöße begehen und in Krisen geraten - nicht häufiger, aber eben auch nicht seltener als die Gesamtgruppe. Ob sie ohne medikamentöse Unterstützung mehr Krisen durchlaufen würden als die übrigen Patienten der Nachsorgeambulanz, lässt sich nur vermuten. Es könnte aber plausibel sein, wenn man davon ausgeht, dass vorwiegend schwere Fälle mediziert werden. Die medikamentöse Therapie ist daher in jedem Fall ein wichtiger Baustein in der forensischen Nachsorgebehandlung. Jedoch kommen anderen Therapieformen wie der psycho- und soziotherapeutischen Behandlung im ambulanten Setting eine mindestens ebenso große Bedeutung zu.

$\mathrm{Zu}$ den Limitationen der vorliegenden Untersuchung ist festzuhalten, dass nur eine eher kleine Fallzahl von insgesamt 117 Personen betrachtet wurde. Auch waren die Fälle stark vorselektiert, was durch die Zuweisung zu einer forensischen Nachsorge zwangsläufig geschieht. Dennoch denken wir, dass die Ergebnisse der Untersuchung einen guten Einblick in die Praxis der medikamentösen Behandlung von Menschen mit Persönlichkeitsstörungen in der forensischen Nachsorge geben. Eine interessante Fragestellung für die Zukunft wäre hier, die Rückfallhäufigkeit bei Personen mit Persönlichkeitsstörung (mediziert vs. nichtmediziert) zu eruieren.

Zusammenfassend lässt sich aus den Erfahrungen an der FTA Berlin die Schlussfolgerung ziehen, dass eine psycho- pharmakologische Behandlung (gegebenenfalls in Form einer Kombination aus Antiandrogenen und Psychopharmaka) im Rahmen des Gesamtbehandlungsplans in der forensischen Nachsorge eine wirksame Unterstützung von Patienten mit Persönlichkeitsstörung bedeutet.

Förderung Das Projekt wurde aus Mitteln der Forensisch-Therapeutischen Ambulanz finanziert.

Funding Open Access funding enabled and organized by Projekt DEAL.

Interessenkonflikt T. Voß, D. Calvano und J. Vogel geben an, dass kein Interessenkonflikt besteht.

Open Access Dieser Artikel wird unter der Creative Commons Namensnennung 4.0 International Lizenz veröffentlicht, welche die Nutzung, Vervielfältigung, Bearbeitung, Verbreitung und Wiedergabe in jeglichem Medium und Format erlaubt, sofern Sie den/die ursprünglichen Autor(en) und die Quelle ordnungsgemäß nennen, einen Link zur Creative Commons Lizenz beifügen und angeben, ob Änderungen vorgenommen wurden.

Die in diesem Artikel enthaltenen Bilder und sonstiges Drittmaterial unterliegen ebenfalls der genannten Creative Commons Lizenz, sofern sich aus der Abbildungslegende nichts anderes ergibt. Sofern das betreffende Material nicht unter der genannten Creative Commons Lizenz steht und die betreffende Handlung nicht nach gesetzlichen Vorschriften erlaubt ist, ist für die oben aufgeführten Weiterverwendungen des Materials die Einwilligung des jeweiligen Rechteinhabers einzuholen.

Weitere Details zur Lizenz entnehmen Sie bitte der Lizenzinformation auf http://creativecommons.org/licenses/by/4.0/deed.de.

\section{Literatur}

Benkert O, Hippius H (2021) Kompendium der psychiatrischen Pharmakotherapie, 13. Aufl. Springer, Berlin

Felthous A, Stanford M, Saß H (2018) Zur Pharmakotherapie impulsiver Aggression bei antisozialen und psychopathischen Störungen. Forens Psychiatr Psychol Kriminol 12:266-278

Freese R, Schmidt-Quernheim F (2014) Mindeststandards forensischer Nachsorge. Qualitätskriterien in forensisch-psychiatrischen Nachsorgeambulanzen. Forens Psychiatr Psychol Kriminol 8:191-198

Gunderson J, Herpertz S, Skodol A, Torgersen S, Zanarini M (2018) Borderline personality disorder. Nat Rev Dis Primers 4:18029

Hancock-Johnson E, Griffiths C, Picchioni M (2017) A focused systematic review of pharmacology treatment for borderline personality disorder. CNS Drugs 31(5):345-356

Hauser N, Herpertz S, Habermeyer E (2021) Das überarbeitete Konzept der Persönlichkeitsstörungen nach ICD-11: Neuerungen und mögliche Konsequenzen für die forensisch-psychiatrische Tätigkeit. Forens Psychiatr Psychol Kriminol 15:31-36

Hörburger TA, Habermeyer E (2020) Zu den Zusammenhängen zwischen paraphilen Störungen, Persönlichkeitsstörungen und Sexualdelinquenz. Forens Psychiatr Psychol Kriminol 14:149-157

Howner K, Andine P, Engbert G, Ekström EH, Lindström E, Nilsson M, Radovic S, Hultcranz M (2020) Pharmacological treatment in forensic psychiatry-A systematic review. Front Psychiatry 10:963

Koch J, Modesitt T, Palmer M, Ward S, Martin B, Wyatt R, Thomas C (2016) Review of pharmacologic treatment in cluster A personality disorders. Ment Health Clin 6(2):75-81

Lau S, Voß T, Mauzaite A, Huchzermeier C, Konrad N, Basdekis-Josza R (2019) Zur Situation stationärer forensischer Psychotherapie 
in Deutschland - Ergebnisse einer Umfrage in Maßregelvollzugseinrichtungen. Psychiatr Prax 46:1-5

Saß H (1986) Psychopathie - Soziopathie - Dissozialität. Zur Differentialtypologie der Persönlichkeitsstörungen. Springer, Berlin

$\mathrm{Saß} \mathrm{H}$ (2006) Biographie, Persönlichkeit und Verantwortung. In: Schneider F (Hrsg) Entwicklungen der Psychiatrie. Springer, Berlin, Heidelberg

Schwarze C, Voß T, Kliesch O, Bauer A, Braunisch S, Feil M (2018) Qualitätskriterien forensischer Ambulanzen des Strafvollzugs. Forens Psychiatr Psychol Kriminol 12:369-379
Stoffers J, Lieb K (2015) Pharmacotherapy for borderline personality disorder-current evidence and recent trends. Curr Psychiatry Rep 17(1):534

Stoffers J, Völlm B, Rücker G, Timmer A, Huband N, Lieb K (2010) Pharmacological interventions for borderline personality disorder. Cochrane Database Syst Rev. https://doi.org/10.1002/14651858

Turner D, Briken P (2018) Treatment of paraphilic disorders in sexual offenders or men with the risk of sexual offending with LHRH agonists. J Sex Med 15(1):77-93

Witzel J (2016) Kombinationen in der Behandlung forensischer Patienten. In: Messer T, Schmauß M (Hrsg) Polypharmazie in der Behandlung psychischer Erkrankungen. Springer, Wien 